\title{
Multiple cutaneous and intestinal metastases in lung cancer: A case report
}

\author{
SHOUTANG LU ${ }^{1 *}, J_{A N S H U ~ Y A N G}{ }^{2,3 *}$, YANLAI SUN ${ }^{2}$ and ZHONGFA XU $U^{1,2}$ \\ ${ }^{1}$ Department of Gastrointestinal Surgery, Affiliated Hospital of Shandong Academy of Medical Sciences, \\ Jinan, Shandong 250031; ${ }^{2}$ Department of Colorectal Cancer Surgery, Shandong Cancer Hospital, Jinan, \\ Shandong 250117; ${ }^{3}$ Shandong Academy of Medical Sciences, Jinan, Shandong 250062, P.R. China
}

Received April 2, 2014; Accepted November 27, 2014

DOI: $10.3892 / 01.2015 .2893$

\begin{abstract}
Lung cancer is a common malignant neoplasm that is prone to distant metastasis. However, the incidence of multiple cutaneous and intestinal metastases is rare. The present study describes the case of a 62-year-old female who was admitted to The Affiliated Hospital of Shandong Academy of Medical Sciences in August 2013 with multiple cutaneous lumps. Contrast-enhanced computed tomography showed nodules and masses in the right lung, and multiple enlarged lymph nodes in the mediastinum and right hilum. Biopsies of the lumps in the right lung and skin revealed moderately-differentiated adenocarcinoma, which were considered to be cutaneous metastases of lung cancer. The patient subsequently experienced symptoms of rectal irritation. A digital rectal examination and colonoscopy were performed, and the consequent pathological biopsy identified moderately-differentiated adenocarcinoma. After analyzing the results of previous pathological examinations and immunohistochemistry, it may be suggested that intestinal metastasis had developed. This case highlights the fact that a comprehensive analysis and examination should be performed for suspected cutaneous and intestinal lesions, during which, a pathological biopsy is of great importance in order to form the correct diagnosis for timely treatment.
\end{abstract}

Correspondence to: Professor Zhongfa Xu, Department of Gastrointestinal Surgery, Affiliated Hospital of Shandong Academy of Medical Sciences, 38 Wuyingshan Road, Jinan, Shandong 250031, P.R. China

E-mail: xuzhongfa2216@sina.com

Dr Yanlai Sun, Department of Colorectal Cancer Surgery, Shandong Cancer Hospital, 440 Jiyan Road, Jinan, Shandong 250117, P.R. China

E-mail: sunyanlai@126.com

${ }^{*}$ Contributed equally

Key words: lung cancer, cutaneous metastasis, intestinal metastasis, pathological biopsy

\section{Introduction}

Lung cancer is one of the most common tumors globally; it is highly malignant and has a high rate of distant metastasis (1). In total, $\sim 50 \%$ of these patients already have distant metastasis when diagnosed, with the most common metastasis sites being the lungs, liver, bone, brain and adrenal glands (1). Cutaneous metastasis of lung cancer is rare, its pathogenesis is by either lymphovascular invasion or hematogenous metastasis (2). The histology of cutaneous metastsis most commonly reveals adenocarcinoma, then squamous/small-cell carcinoma, followed by large-cell carcinoma (3). Common treatment modalities include surgery, chemotherapy and radiotherapy. Currently, the prognosis for patients with cutaneous metastasis of lung cancer is poor. Intestinal metastases from lung cancer are rare and the diagnosis is often late, with clinical symptoms of bowel occlusion and intestinal bleeding (4). In certain cases the clinical manifestations of the metastases have been observed prior to those of the primitive tumour (5). However, in the presence of bowel occlusion and intestinal bleeding of uncertain origin, obtaining a clinical history is particularly important and diagnostic procedures must be performed to rule out a secondary pathology (6). Until now, simultaneous cutaneous and intestinal metastases have never been reported. The present study reports such a case that was recently admitted to The Affiliated Hospital of Shandong Academy of Medical Sciences (Jinan, China). Written informed consent was obtained from the patient.

\section{Case report}

A 62-year-old female was admitted to The Affiliated Hospital of Shandong Academy of Medical Sciences in August 2013 with multiple lumps in the right thigh, armpit and scalp that had been present for one month. A number of these lumps had ulcerated two weeks prior to the visit. Three lumps were observed on the scalp, among which the top lump was the largest. This lump was a hard, $3 \times 2 \mathrm{~cm}$ protrusion, which was recessed and ulcerated at the center, with a clear embankment-like boundary. The other two bulges looked like craters, with clear boundaries and no ulceration or exudation. No tenderness was reported. In addition, a furuncle-like lump was found on the right thigh, 


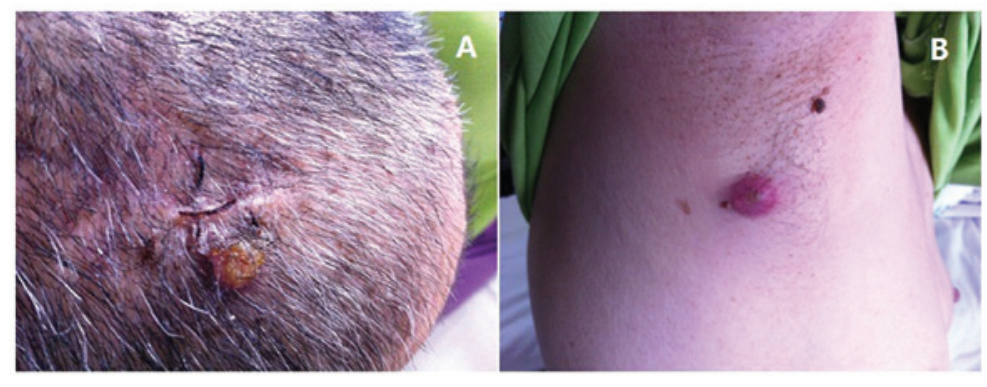

Figure 1. Clinical photograph showing (A) a lump on the top of the head and (B) a purple, protruding lump in the right armpit.

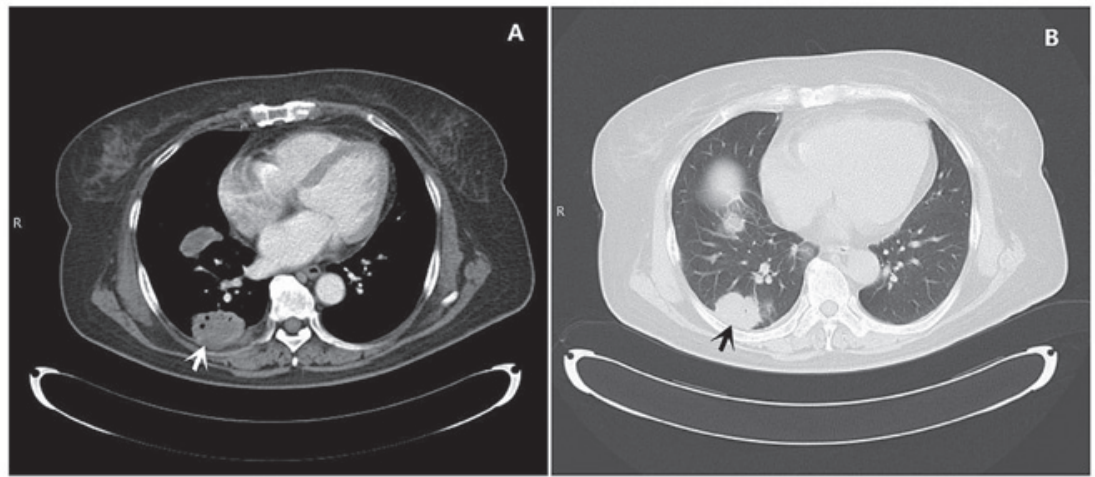

Figure 2. Chest computed tomography showing the primary tumor in the inferior lobe of the right lung (arrow) in the (A) mediastinal and (B) pulmonary windows.

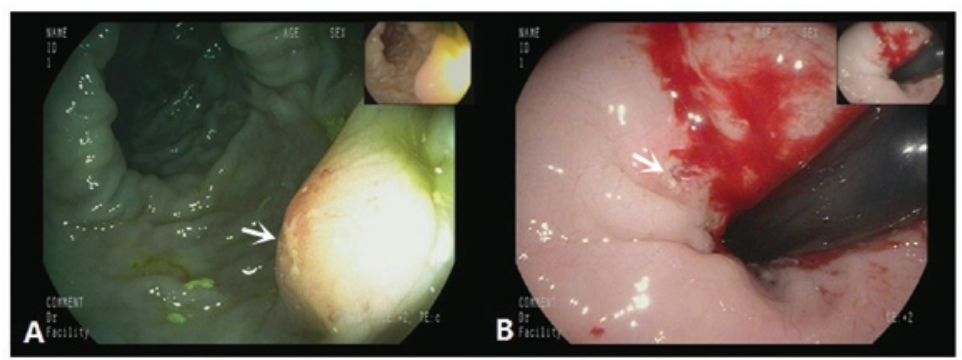

Figure 3. Electronic colonoscopy images showing (A) a $0.3 \times 0.3-\mathrm{cm}$ lump in the transverse colon that exhibited a rough mucosal membrane on the top, with clear boundaries and (B) an ulcer with a diameter of $\sim 1 \mathrm{~cm}$, a recessed center, a peripheral bulge and a hard texture on the rectum.

which was swollen and ulcerated, with mild tenderness. A purple, protruding $2 \times 2-\mathrm{cm}$ lump could also be observed in the right armpit, with furuncle-like embossing of the top and clear boundaries. The lump was of moderate texture, with a certain degree of tenderness (Fig. 1). Upon physical examination, chest auscultation revealed clear breathing sounds for the left lung, while those of the right lung were comparatively lower. There was no rhonchus or moist rale and other parameters were normal. Chest computed tomography $(\mathrm{CT})$ showed nodules and masses in the right lung, with multiple enlarged lymph nodes in the mediastinum and right hilum (Fig. 2). This suggested a diagnosis of primary right lung cancer with intrapulmonary and lymphatic metastases in the mediastinum and right hilum. Resection of the tumors on the scalp, right thigh and armpit was performed due to the ulcerated cutaneous nature of the tumors. Pathological examination showed moderately-differentiated adenocarcinoma, which was considered to be metastatic cancer. A percutaneous biopsy of the right lung tumor showed moderately-differentiated adenocarcinoma. During the hospitalization period, the patient experienced increased stool frequency without obvious cause, which included tenesmus with blood and pus, but no abdominal pain, nausea or vomiting. A digital rectal examination revealed blood and a $4 \times 3-\mathrm{cm}$ lump at the rear of the perineal area, which was compressing the rectum. Colonoscopy showed a $0.3 \times 0.3-\mathrm{cm}$ lump on the inside of the transverse colon, which exhibited a rough mucosal membrane on the top, with clear boundaries. An ulcer with a diameter of $\sim 1 \mathrm{~cm}$, a recessed center, a peripheral bulge and a hard texture was observed on the rectum (Fig. 3). Biopsies were taken from the two sites, which were both subsequently diagnosed as moderately-differentiated adenocarcinoma. A similar pathology as that shown on light microscopy (Fig. 4) and similar immunohistochemistry results (Table I) indicated that the tumors in the intestines, scalp and thigh were all metastases of the primary lung cancer. The patient is currently undergoing systemic chemotherapy with intravenous gemcitabine $(1.4 \mathrm{~g}$, days 1 
Table I. Immunohistochemistry results of primary tumors and corresponding metastases.

\begin{tabular}{|c|c|}
\hline Primary and metastatic sites & Immunohistochemistry result \\
\hline Primary lung cancer & CK7(+); CDX2 spotty(+); CK20(-); TTF-1(-); vimentin(-) \\
\hline Cutaneous metastasis & $\begin{array}{l}\text { CK7(+); CK19(+); CA19-9 spotty(+); CDX2 scattered(+); CK20(-); TTF-1(-); } \\
\text { GCDFP-15(-); ER(-); PR(-) }\end{array}$ \\
\hline Rectal metastasis & CK7(+); CK19(+); CDX2 spotty(+); CK20(-); TTF-1(-) \\
\hline Transverse colon metastasis & CK7(+); CK19(+); CDX2 spotty(+); CK20(-); TTF-1(-); villin(-) \\
\hline
\end{tabular}

CK, cytokeratin; CDX2, caudal-type homeobox 2; TTF-1, thyroid transcription factor 1; CA19-9, cancer antigen 19-9; GCDFP-15, gross cystic disease fluid protein 15; ER, estrogen receptor; PR, progesterone receptor.

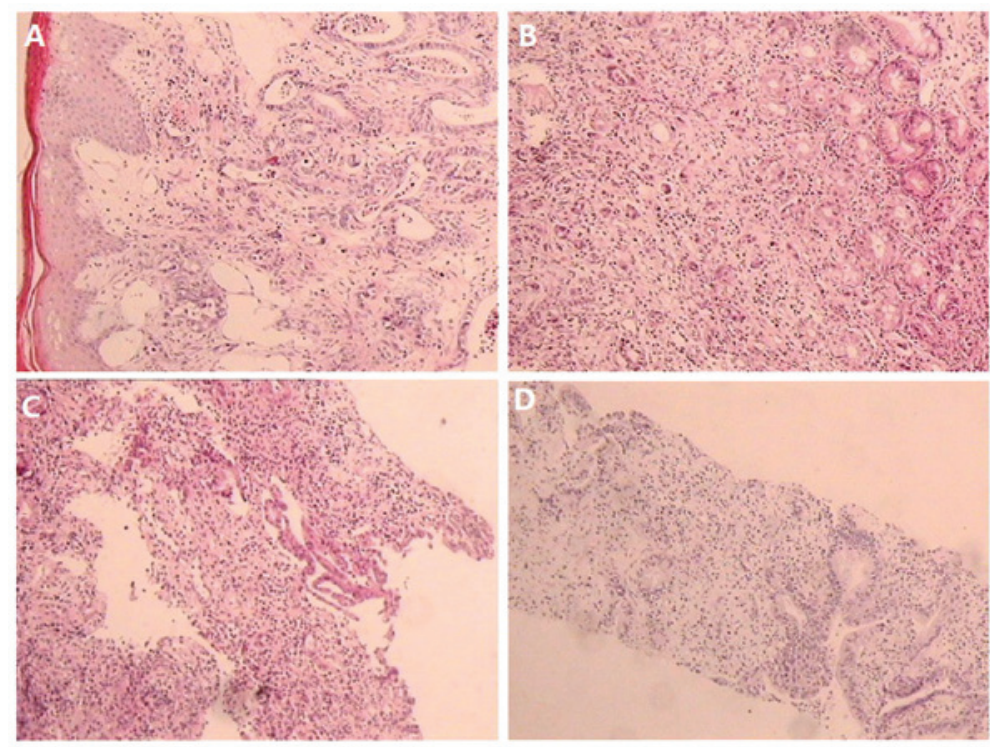

Figure 4. Histopathology of (A) cutaneous metastasis, (B) rectal metastasis, (C) transverse colon metastasis and (D) the primary tumor of the right lung. All were histopathologically diagnosed as moderately-differentiated adenocarcinoma (hematoxylin and eosin; original magnification, x100).

and 8) and cisplatin (40 mg, days 1-3) every two weeks. At the time of writing, the patient had undergone eight weeks of a six month treatment. However, as the patient exhibits multiple cutaneous metastases and intestinal metastasis, the expected surival time is poor.

\section{Discussion}

More than half of all lung cancers have metastasized when diagnosed. These metastases occur most often in the thoracic lymph nodes (46-85\%), pleura (14-46\%), brain (14-45\%), adrenal gland (36-64\%), bone (21-41\%), contralateral lung (13-43\%) and kidney (13-43\%), and a small percentage of metastases are in the abdominal lymph nodes, spleen, pancreas, heart, pericardium and other regions $(1,7)$. Cases of lung cancer with cutaneous and intestinal metastases are rare.

Cutaneous metastasis is caused by primary cancer-derived cells that grow in the skin (8). According to the published literature, the incidence of cutaneous metastasis is $2.9-5.3 \%$ in general (9), and $1-12 \%$ for lung cancer (10). Clinically, cutaneous metastasis often manifests as single or multiple nodules, possibly at multiple sites (usually close to the primary tumor), which differ in size (often $0.5-10 \mathrm{~cm}$ ), and are round or oval, hard, relatively immobilized, normally colored, purple or bright red, and ulcerated or cauliflower-like, with bleeding in certain cases $(8,11)$. Certain cases may also manifest as erysipelas-like cancer, vascular dilation or bullous-like lesions, papules, plaques or scarring (12). For the majority of cases, cutaneous metastasis occurs during the progression of primary tumor following the initial diagnosis, but for a few cases, cutaneous metastasis is found prior to the primary tumor or simultaneously with the latter $(7,10)$. Cutaneous metastasis of the breast and oral cancers often result from hematogenous and lymphatic metastasis, where the latter pathway is pivotal, while for other tumors, hematogenous metastasis is usually the primary cause. Metastasis through the lymphatic pathway may explain why cutaneous metastasis occurs proximal to the primary tumor (13). Lung cancer-derived cutaneous metastasis cannot be differentiated from cutaneous metastases of other sources based on the gross specimen. Lung cancer-derived cutaneous metastases are most commonly from lung adenocarcinoma, followed by squamous cell carcinoma, small cell lung cancer and large cell lung cancer (14). Lung adenocarcinoma is usually derived from bronchial epithelial goblet 
cells, usually the borderline type, and is often asymptomatic in the early stages and not diagnosed till the later stages when metastasis or compression symptoms occur. The prognosis of cutaneous metastasis of lung cancer is poor, and despite the use of chemoradiotherapy, the median survival time is only 3-6 months (11).

Intestinal metastasis of lung cancer is even rarer than cutaneous metastasis, with an incidence of $\sim 0.19 \%$ according to the published literature (15). Intestinal metastasis causes abdominal symptoms such as abdominal pain, bloating, bowel dysfunction and bleeding. CT or even positron emission tomography-CT examinations rarely detect small metastases, and false-negative results are common. Patients may initially present with intestinal symptoms rather than typical signs of primary lung cancer due to a lack of specific symptoms. The intestinal metastasis diagnosed by colonoscopy may be misdiagnosed as the primary tumor, therefore a biopsy is required for an accurate diagnosis (16). The path of the intestinal metastasis of lung cancer is currently unclear, although it is generally considered to be lymphatic or hematogenous. However, from previous clinical experience we propose the following two possibilities: i) Metastasis may have occurred through the paravertebral venous system to the intestinal mucosa; or ii) since the patient had a long-term cough with sputum, the cancer cells may have be coughed up with the sputum and swallowed into the digestive tract, where they adhered to the intestines and became established as metastasis. As the patient in the current report exhibited no obvious cough, intestinal metastasis was potentially the result of lymphatic and hematogenous metastaiss. There is currently no solid evidence to support these hypotheses, so relevant basic research is required. Intestinal metastasis indicates an advanced grade of lung cancer, which leaves palliative treatment and supportive care as the only treatment options (17). The median survival time is only 4-8 weeks after the diagnosis of intestinal metastasis of lung cancer (18).

The patient in the present study exhibited no obvious cough or any abnormal lung-related signs or symptoms, and originally presented with multiple cutaneous lumps whose characteristics were similar to that reported by the literature. The primary cancer was identified during the CT examination, and intestinal metastasis was detected by digital rectal examination, colonoscopy and biopsy due to rectal irritation. In summary, the patient exhibited multiple cutaneous and intestinal metastases, possibly the additive result of lymphatic and hematogenous metastasis. The diagnosis of this case was quick, and the patient is currently undergoing systemic chemotherapy with gemcitabine plus cisplatin. As the patient already exhibits multiple cutaneous metastases and intestinal metastasis, the expected survival time may not be long. The efficacy and prognosis of the treatment requires further observation and analysis.

In conclusion, lung cancer is highly malignant and prone to distant metastases, however, cutaneous and intestinal metastases are rare. The present patient originally presented with cutaneous metastases, and the primary tumor and intestinal metastasis was only found during the examination, after which systemic chemotherapy was administered. For suspected cutaneous and intestinal lesions, a comprehensive analysis and examination should be performed, including a timely pathological biopsy according to the characteristics of the cutaneous lesion and a digital rectal examination. A colonoscopy plus biopsy should be routinely used for intestinal lesions to obtain an accurate diagnosis, so that the correct treatment can be applied quickly and patient survival can be prolonged.

\section{References}

1. Jemal A, Bray F, Center MM, et al: Global cancer statistics. CA Cancer J Clin 61: 69-90, 2011.

2. Alkhayat $\mathrm{H}$ and Hong $\mathrm{CH}$ : Cutaneous metastases from non-small cell lung cancer. J Cutan Med Surg 10: 304-307, 2006.

3. Song Z, Lin B, Shao L and Zhang Y: Cutaneous metastasis as a initial presentation in advanced non-small cell lung cancer and its poor survival prognosis. J Cancer Res Clin Oncol 138: 16131617, 2012.

4. Nishizawa Y, Kobayashi A, Saito N, et al: Surgical management of small bowel metastases from primary carcinoma of the lung. Surg Today 42: 233-237, 2012.

5. Berger A, Cellier C, Daniel C, et al: Small bowel metastases from primary carcinoma of the lung: clinical findings and outcome. Am J Gastroenterol 94: 1884-1887,1999.

6. Cipollone G, Santarelli G, Quitadamo S, et al: Small bowel metastases from lung cancer. Chir Ital 56: 639-648, 2004 (In Italian).

7. Jemal A, Center MM, DeSantis C and Ward EM: Global patterns of cancer incidence and mortality rates and trends. Cancer Epidemiol Biomarkers Prev 19: 1893-1907, 2010.

8. Riahi RR and Cohen PR: Clinical manifestations of cutaneous metastases. Am J Clin Dermatol 13: 103-112, 2012.

9. Krathen RA, Orengo IF and Rosen T: Cutaneous metastasis: a meta-analysis of data. South Med J 96: 164-167, 2003.

10. Mollet TW, Garcia CA and Koester G: Skin metastases from lung cancer. Dermatol Online J 15: 1, 2009.

11. Triller Vadnal K, Triller N, Pozek I, et al: Skin metastases of lung cancer. Acta Dermatovenerol Alp Pannonica Adriat 17: 125-128, 2008.

12. Inamadar AC, Palit A, Athanikar SB, et al: Inflammatory cutaneous metastasis as a presenting feature of bronchogenic carcinoma. Indian J Dermatol Venereol Leprol 69: 347-349, 2003.

13. Pathak S, Joshi SR, Jaison J and Kendre D: Cutaneous metastasis from carcinoma of lung. Indian Dermatol Online J 4: 185-187, 2013.

14. Sha D, Wang C and Wang W: Skin metastasis of lung cancer: A clinical analysis and review of literature. Central China Medical Journal 33: 57-59, 2009.

15. Kim MS, Kook EH, Ahn SH, et al: Gastrointestinal metastasis of lung cancer with special emphasis on a long-term survivor after operation. J Cancer Res Clin Oncol 135: 297-301, 2009.

16. Yang CJ, Hwang JJ, Kang WY, et al: Gastro-intestinal metastasis of primary lung carcinoma: clinical presentations and outcome. Lung Cancer 54: 319-323, 2006.

17. McNeill PM, Wagman LD and Neifeld JP: Small bowel metastases from primary carcinoma of the lung. Cancer 59: 1486-1489, 1987.

18. John AK, Kotru A and Pearson HJ: Colonic metastasis from bronchogenic carcinoma presenting as pancolitis. J Postgrad Med 48: 199-200, 2002. 